\title{
MicroRNA-33b suppresses the proliferation and metastasis of hepatocellular carcinoma cells through the inhibition of Sal-like protein 4 expression
}

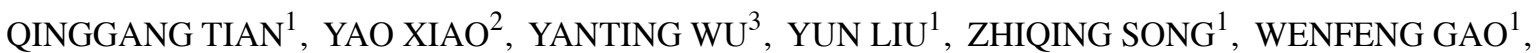 \\ JING ZHANG ${ }^{1}$, JINGLING YANG ${ }^{1}$, YUGUO ZHANG ${ }^{1}$, TUANKUI GUO ${ }^{1}$, FURONG DAI ${ }^{1}$ and ZHIGANG SUN ${ }^{4}$ \\ ${ }^{1}$ Department of General Surgery, The 4th Affiliated Hospital of Baotou Medical College, Baotou, Inner Mongolia 014040; \\ ${ }^{2}$ Department of Hepatobiliary and Pancreatic Surgery, Xiangya Hospital, Central South University, Changsha, \\ Hunan 410008; ${ }^{3}$ Department of General Surgery, The Third Clinical Medical School of Inner Mongolia Medical University \\ (Baogang Hospital of Inner Mongolia), Baotou, Inner Mongolia 014010; ${ }^{4}$ Department of Neurosurgery, \\ Baotou Eighth Hospital, Baotou, Inner Mongolia 014040, P.R. China
}

Received October 4, 2015; Accepted September 16,2016

DOI: $10.3892 /$ ijmm.2016.2754

\begin{abstract}
MicroRNAs (miRNAs or miRs) have been found to participate in the development and malignant progression of human cancers by negatively mediating the expression of their target genes. Recently, miR-33b has been reported to be involved in multiple types of human cancer, including hepatocellular carcinoma (HCC). However, the underlying regulatory mechanisms of miR-33b in HCC cell growth and metastasis remain largely unclear. In the present study, RT-qPCR revealed that miR-33b was significantly downregulated in HCC tissues compared to their matched adjacent normal tissues. Moreover, the miR-33b level was significantly lower in advanced-stage HCC (stages T3-T4) compared to early-stage HCC (stages T1-T2). Furthermore, it was also downregulated in the HCC cell lines, LH86, HepG2, LMH and PLHC-1, when compared with the THLE-3 normal human liver cells. We further demonstrated that the overexpression of miR-33b led to a significant decrease in the proliferation, migration and invasion of HepG2 and LH86 cells. Luciferase reporter assay identified Sal-like protein 4 (SALL4) as a target gene of miR-33b, and its protein expression was negatively regulated by miR-33b in HepG2 and LH86 cells. Moreover, the restoration of SALL4 expression markedly reversed the inhibitory effect of miR-33b overexpression on the proliferation, migration and invasion of HepG2 and LH86 cells, indicating that SALL4 is involved in miR-33b-mediated malignant phenotypes of HCC cells. Furthermore, we found that SALL4 was significantly upregulated in HCC tissues compared to their matched adjacent normal tissues, and its increased expression
\end{abstract}

Correspondence to: Professor Zhigang Sun, Department of Neurosurgery, Baotou Eighth Hospital, South Gate Outside Street 22, Baotou, Inner Mongolia 014040, P.R. China

E-mail: sunzhigang1818@sina.com

Key words: hepatocellular carcinoma, microRNA-33b, Sal-like protein 4 , proliferation, migration, invasion was significantly associated with the advanced malignancy of HCC. Moreover, SALL4 was also upregulated in HCC cell lines compared to the THLE-3 normal human liver cells. Finally, we found that the SALL4 expression inversely correlated with the miR-33b level in HCC tissues. On the whole, the findings of our study demonstrate that miR-33b suppresses the proliferation and metastasis of HCC cells through the inhibition of SALL4 expression. Therefore, miR-33b/SALL4 may become a potential therapeutic target for the treatment of HCC.

\section{Introduction}

Hepatocellular carcinoma (HCC) is one of the most common human cancers, the incidence of which is increasing rapidly (1). Since HCC is often diagnosed at an advanced stage, it has become a leading cause of cancer-related mortality (2). Genetic and environmental alterations have been identified as two leading factors during HCC pathogenesis $(3,4)$. Moreover, deregulations of oncogenes or tumor suppressors have been found in $\mathrm{HCC}$, and the detailed roles of genetic and epigenetic factors have been extensively investigated, which may aid in the development of effective diagnostic and therapeutic strategies for HCC (5).

Sal-like protein 4 (SALL4), a zinc finger transcription factor, has been identified as an important marker for stem cells, and is involved in the maintenance of self-renewal in embryonic stem cells (6). Moreover, SALL4 has been found to be mainly expressed in fetal livers and has been used as a marker of several human cancers, including HCC $(7,8)$. Recently, SALL4 was found to be upregulated in HCC (9), and to promote tumorigenesis and the malignant progression of HCC (10-12). Therefore, SALL4 may become a promising target for the treatment of HCC. However, to date, the regulatory mechanisms of action of SALL4 in HCC remain largely unknown.

MicroRNAs (miRNAs or miRs) are a class of non-coding RNAs, 18-25 nucleotides in length, that can induce mRNA degradation or suppress protein translation by binding to the 3' untranslated regions (3'UTRs) of mRNAs of specific genes (13). Moreover, various miRNAs have been reported 
to play promoting or suppressive roles in human cancers by negatively mediating their target genes, which act as tumor suppressors or oncogenes $(14,15)$. Therefore, revealing the roles and regulatory mechanisms of miRs seems to be important for cancer treatment. A variety of miRNAs have been found to be deregulated in HCC, such as miR-122, miR-124, miR-138 and miR-203 (16-19). Recently, miR-33a, a member of the miR-33 family, was found to inhibit HCC cell growth (20). However, the expression, as well as the role of miR-33 in HCC has not been studied previously, at least to the best of our knowledge.

In the present study, we aimed to investigate the expression, as well as the regulatory mechanisms of miR-33b in HCC. Our data demonstrated that miR-33b was significantly downregulated in HCC tissues and cell lines, and that it suppressed the proliferation, migration and invasion of HCC cells by directly targeting SALL4, which was upregulated and inversely correlated with miR-33b in HCC.

\section{Materials and methods}

Tissue collection. This study was approved by the Ethics Committee of the 4th Affiliated Hospital of Baotou Medical College, Baotou, China. A total of 23 primary HCC tissues, as well as their matched normal adjacent specimens were collected from the 4th Affiliated Hospital of Baotou Medical College from December 2013 to June 2014. Written informed consent was obtained from all participants. Among these 23 cases of HCC, 15 were male, and 8 were female; the youngest patient was 38 years of age, and the oldest was 67 years of age, with the average age being 52.4 years. In addition, 4 patients with $\mathrm{HCC}$ were at T1 stage, 6 were at T2 stage, 8 were at T3 stage, and 5 were at T4 stage of the disease. None of the patients had received any pre-operative chemotherapy, radiotherapy, or embolization. The histomorphology of all the samples was confirmed by the Department of Pathology of the 4th Affiliated Hospital of Baotou Medical College). HCC tissues were immediately snap-frozen in liquid nitrogen after surgical removal.

Cell culture. The human HCC cell lines, HepG2, LH86, LMH and PLHC-1, the normal liver cell line, THLE-3, as well as the 293 cells were obtained from the Cell Bank of Baotou Medical College. The cells were cultured in Dulbecco's modified Eagle's medium (DMEM) with $10 \%$ fetal bovine serum (FBS) (both from Life Technologies, Carlsbad, CA, USA) at $37^{\circ} \mathrm{C}$ in a humidified incubator containing $5 \% \mathrm{CO}_{2}$.

Reverse transcription-quantitative $R T-P C R$ (RT-qPCR). Total RNA was extracted from the cells and tissues using TRIzol reagent (Life Technologies), according to the manufacturer's instructions. RNA was then converted into cDNA using the MiRNA Reverse Transcription kit (Life Technologies). Reverse transcription was performed at $16^{\circ} \mathrm{C}$ for $30 \mathrm{~min}$, followed by an incubation at $42^{\circ} \mathrm{C}$ for $30 \mathrm{~min}$ and enzyme inactivation at $85^{\circ} \mathrm{C}$ for $5 \mathrm{~min}$. Quantitative (qPCR) was then performed to examine miR-33b expression using the miRNA qPCR Detection kit (GeneCopoeia, Rockville, MD, USA) on an ABI 7500 thermocycler (Life Technologies). The U6 gene was used as an internal reference. mRNA expression was determined using the SYBR-Green I Real-Time PCR kit (Biomics, Nantong,
China) on an ABI 7500 thermocycler. Glyceraldehyde 3-phosphate dehydrogenase (GAPDH) was used as an internal reference. The sequences of the primers used for PCR were as follows: SALL4 forward, 5'-CCCGGCAGTAAGGACTGTC-3' and reverse, 5'-TCTCTGTCTTTAGGTACACCACA-3'; and GAPDH forward, 5'- ACAACTTTGGTATCGTGGAAGG-3' and reverse, 5'-GCCATCACGCCACAGTTTC-3'. The reaction conditions were $95^{\circ} \mathrm{C}$ for $5 \mathrm{~min}$, followed by 40 cycles of denaturation at $95^{\circ} \mathrm{C}$ for $15 \mathrm{sec}$ and annealing/elongation step at $60^{\circ} \mathrm{C}$ for $30 \mathrm{sec}$. The relative expression was analyzed using the $2^{-\Delta \Delta \mathrm{Ct}}$ method, as previously described (21).

3-(4,5-Dimethylthiazol-2-yl)-2,5-diphenyltetrazolium bromide (MTT) assay. Cell proliferation was determined by MTT assay. The HepG2 and LH86 cells were cultured in 96-well plate, each well with $100 \mu \mathrm{l}$ of fresh serum-free medium with $0.5 \mathrm{gl}$ MTT. Following incubation at $37^{\circ} \mathrm{C}$ for $12,24,48$ and $72 \mathrm{~h}$, the medium was removed by aspiration and $50 \mu \mathrm{l}$ of dimethyl sulfoxide (DMSO) was added to each well. Following incubation at $37^{\circ} \mathrm{C}$ for a further $10 \mathrm{~min}$, the absorbance at $570 \mathrm{~nm}$ (A570) of each sample was measured using a plate reader (Tecan Infinite M200; Tecan Group Ltd., Männedorf, Switzerland).

Wound healing assay. Wound healing assay was used to examine the migratory capacity of the HCC cells. The HepG2 and LH86 cells were cultured to full confluence. Wounds of approximately $1 \mathrm{~mm}$ in width were created using a plastic scriber. The cells were washed and then cultured in DMEM containing $10 \%$ FBS for $48 \mathrm{~h}$. The cells were then observed and photographed under a microscope (CX23; Olympus, Tokyo, Japan).

Transwell assay. The invasive capacity of the HepG2 and LH86 cells was determined in 24-well Transwell chambers (BD Biosciences, Franklin Lake, NJ, USA), which has a layer of Matrigel. A cell suspension containing 5,000 cells was added to the upper chamber, and DMEM containing 10\% FBS was added to the lower chamber. Following incubation for $24 \mathrm{~h}$, non-invading cells, as well as the matrix gel on the interior of the inserts was removed using a cotton-tipped swab. The invasive cells on the lower surface of the membrane were stained with gentian violet (Beyotime Biotechnology, Haimen, China), and then rinsed with water and dried in air. The invasive cells were photographed and the cell number was determined under a microscope (CX23; Olympus).

Transfection. Lipofectamine 2000 (Life Technologies) was used to perform transfection according to the manufacturer's instructions. Briefly, the cells were cultured to $70 \%$ confluence, and resuspended in serum-free medium. Scramble miR (miR-NC), miR-33b mimics, miR-33b inhibitor, the pc-DNA3.1-SALL4 plasmid (all generated by Amspring, Changsha, China) and Lipofectamine 2000 were diluted with serum-free medium. The diluted Lipofectamine 2000 was added to the diluted miR or plasmid, and incubated for $20 \mathrm{~min}$ at room temperature, and then added to the cell suspension. Following incubation at $37^{\circ} \mathrm{C}, 5 \% \mathrm{CO}_{2}$ for $6 \mathrm{~h}$, the medium was replaced with normal serum-containing medium. The cells were then cultured for $24 \mathrm{~h}$ for use in the following assays. Untransfected cells were used as controls. 

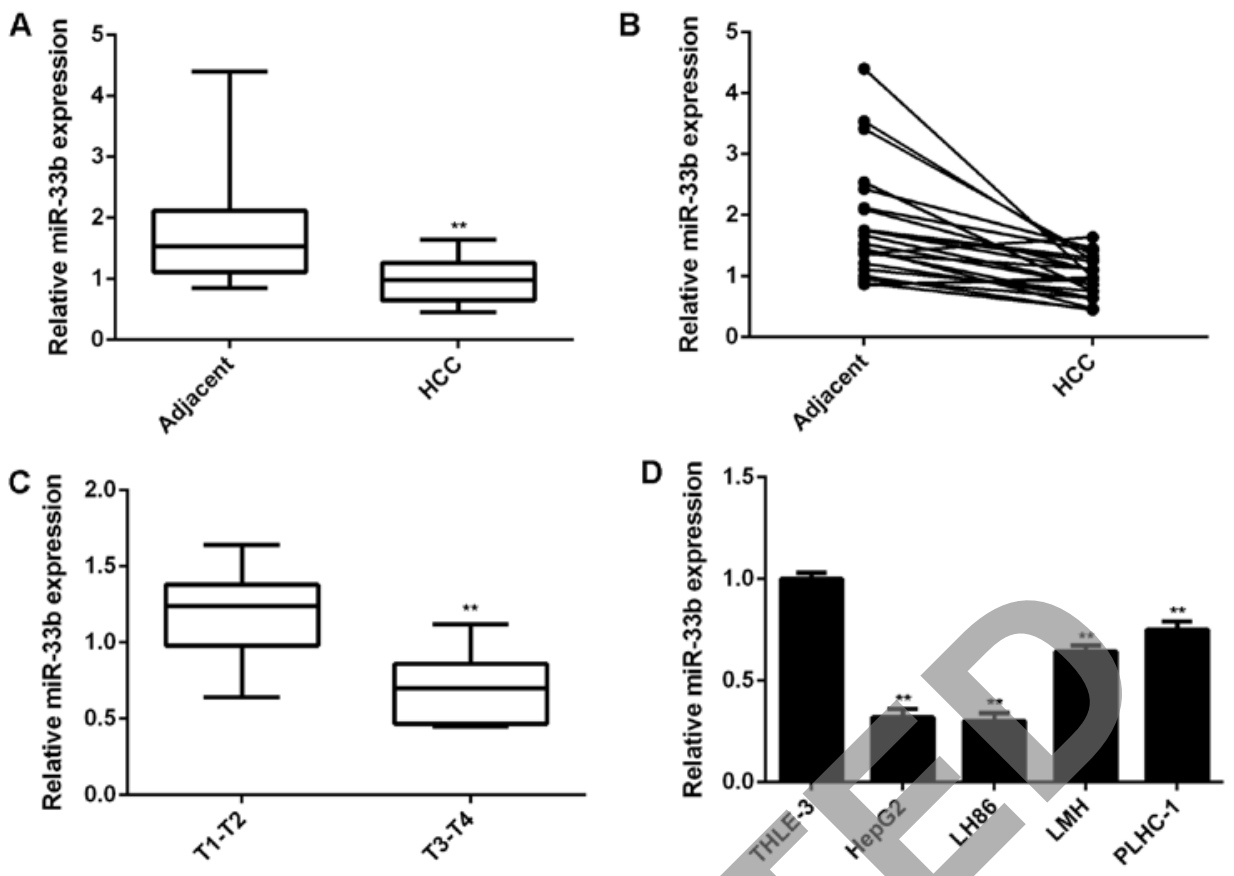

Figure 1. (A and B) RT-qPCR was used to determine the relative expression of miR-33b in hepatocellular carcinoma (HCC) tissues and their matched adjacent normal tissues (adjacent). ${ }^{* *} \mathrm{P}<0.01$ vs. adjacent tissues. (C) RT-qPCR was used to determine the relative expression of miR-33b in the early-stage HCC tissues (stages T1-T2) and the advanced-stage HCC tissues (stages T3-T4). ${ }^{* *} \mathrm{P}<0.01$ vs. stages T1-T2. (D) RT-qPCR was performed to determine the relative expression of miR-33b in the human HCC cell lines, HepG2, LH86, LMH and PLHC-1, and in the normal liver cell line, THLE-3. ${ }^{* *}$ P $<0.01$ vs. THLE-3 cells.

Western blot analysis. The cells were solubilized in cold RIPA lysis buffer (Life Technologies). Proteins were separated by $10 \%$ sodium dodecyl sulfate-polyacrylamide gel electrophoresis (SDS-PAGE), and transferred onto a polyvinylidene difluoride membrane (Life Technologies). The membrane was incubated with phosphate-buffered saline (PBS) containing $5 \%$ milk (Yili, Beijing, China) overnight at $4^{\circ} \mathrm{C}$, which was then incubated with rabbit anti-SALL4 polyclonal antibody (1:50; ab31968), rabbit anti-GAPDH polyclonal antibody (1:50; ab9485), at room temperature for $3 \mathrm{~h}$. After being washed with PBS 3 times, the membrane was incubated with mouse anti-rabbit secondary antibody (1:10,000; ab99697) at room temperature for $1 \mathrm{~h}$. An ECL kit (Pierce Chemical, Rockford, IL, USA) was then used to perform chemiluminence detection according to the manufacturer's instructions. The relative protein expression was represented as the density ratio versus GAPDH.

Bioinformatics analysis. Bioinformatics analysis was performed to predict the putative target genes of miR-33b using Targetscan 3.2 software (www.targetscan.org), according to the manufacturer's instructions.

Dual Luciferase reporter assay. The directed Mutagenesis kit (Stratagene, La Jolla, CA, USA) was used to construct the mutant type (MT) of SALL4 3'UTR lacking complimentarity with the miR-33b seed sequence, in accordance with the manufacturer's instructions. Subsequently, the wild-type (WT) SALL4 3'UTR or MT SALL4 3'UTR was cloned into the psiCHECK-2 vector (Promega, Madison, WI, USA) downstream of the Renilla luciferase gene, respectively. Subsequently, 293 cells were seeded into 48 -well plates $(50,000$ cells/well) and cultured for $24 \mathrm{~h}$. The cells were then co-transfected with the reporter vectors and the miR-33b mimic or miR-NC using Lipofectamine 2000.
Luciferase activity was measured at $48 \mathrm{~h}$ post-transfection using the Dual-Luciferase Reporter assay system (Promega) on an Lmax multiwell luminometer (Molecular Devices, Sunnyvale, CA, USA), in accordance with the manufacturer's instructions.

Statistical analysis. Data are expressed as the means \pm SD. Statistical analysis was performed using SPSS 17.0 software (SPSS, Armonk, NY, USA). The statistical correlation of data between groups was analyzed by one-way analysis of variance (ANOVA). A value of $\mathrm{P}<0.05$ was considered to indicate a statistically significant difference.

\section{Results}

miR-33b is downregulated in HCC. To elucidate the role of miR-33b in HCC, we first conducted RT-qPCR to determine its expression levels in HCC tissues and cell lines. The expression levels of miR-33b were markedly decreased in the HCC tissues compared to their matched adjacent tissues $(\mathrm{P}<0.01$; Fig. $1 \mathrm{~A}$ and $\mathrm{B}$ ). Moreover, our data demonstrated that the miR-33b level was markedly lower in the advanced-stage HCC tissues (stages (T3-T4 stages) compared to the early-stage HCC tissues (stages $(\mathrm{T} 1-\mathrm{T} 2)(\mathrm{P}<0.01)$, suggesting that the decreased expression of miR-33b was significantly associated with the malignant progression of HCC (Fig. 1C). We further demonstrated that miR-33b was significantly downregulated in the HCC cell lines, HepG2, LH86, LMH and PLHC-1, when compared to the normal liver cell line, THLE-3 (Fig. 1D; P<0.01). Therefore, miR-33b is downregulated in HCC.

Overexpression of miR-33b suppresses the proliferation, migration and invasion of HCC cells. As the HepG2 and LH86 cell lines showed the most significant decrease in miR-33b 
A

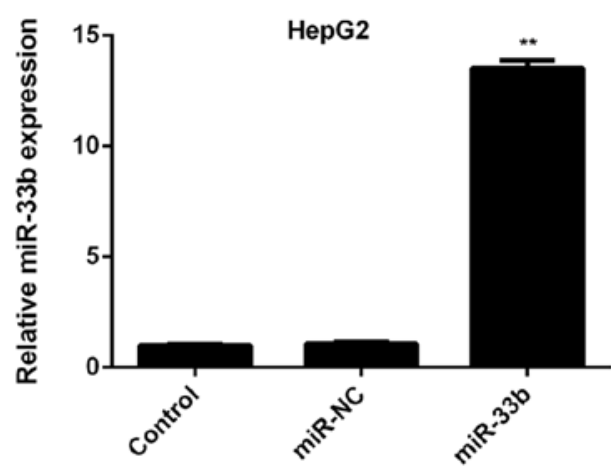

B

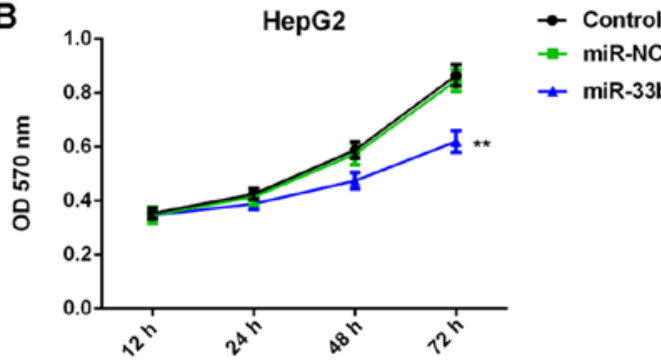

C
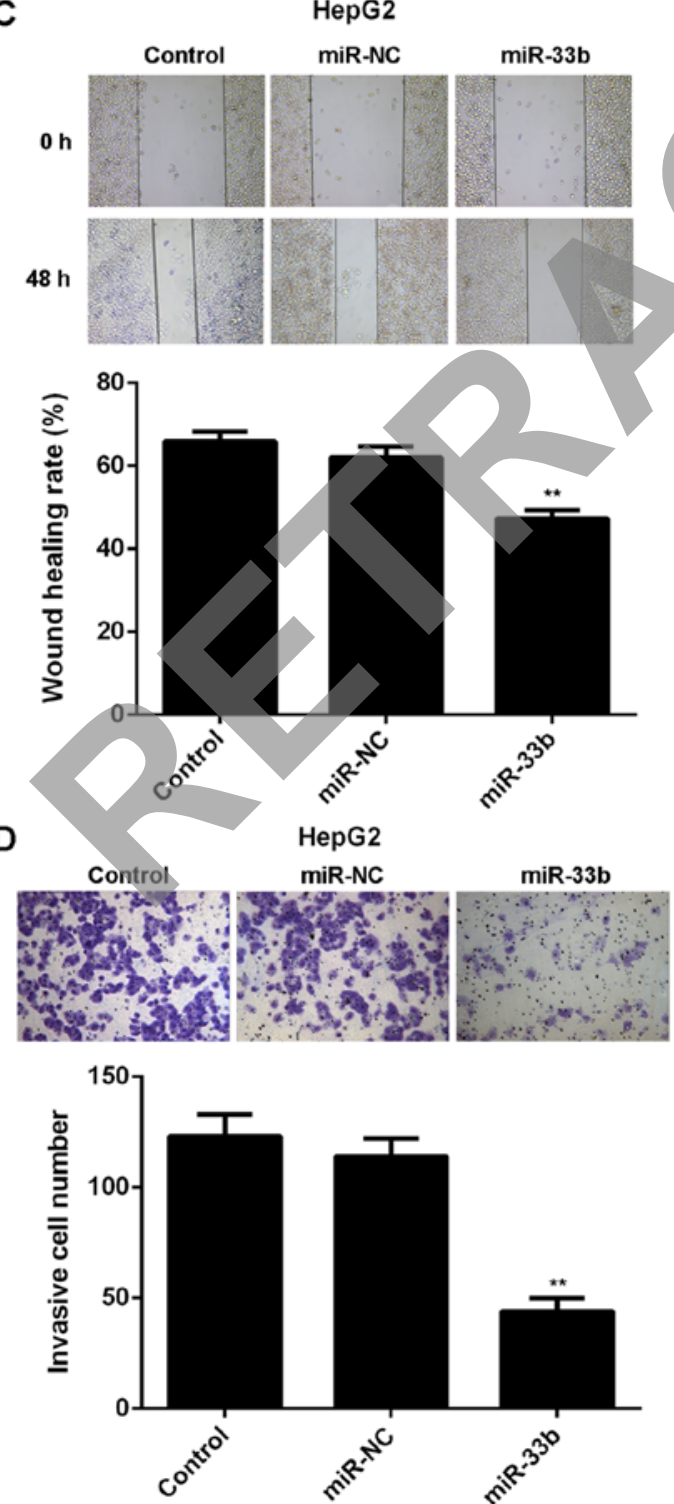
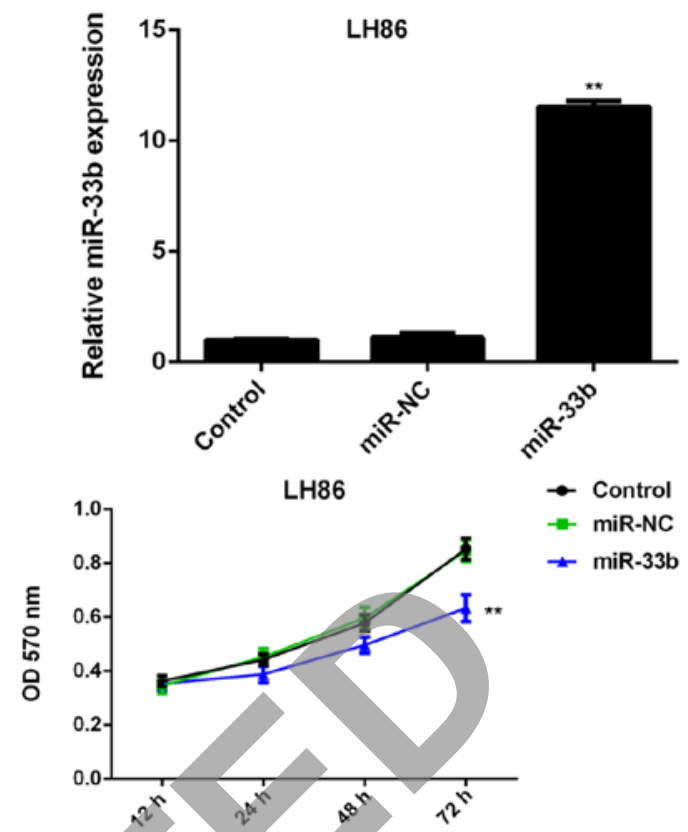

H86 miR-NC miR-33b
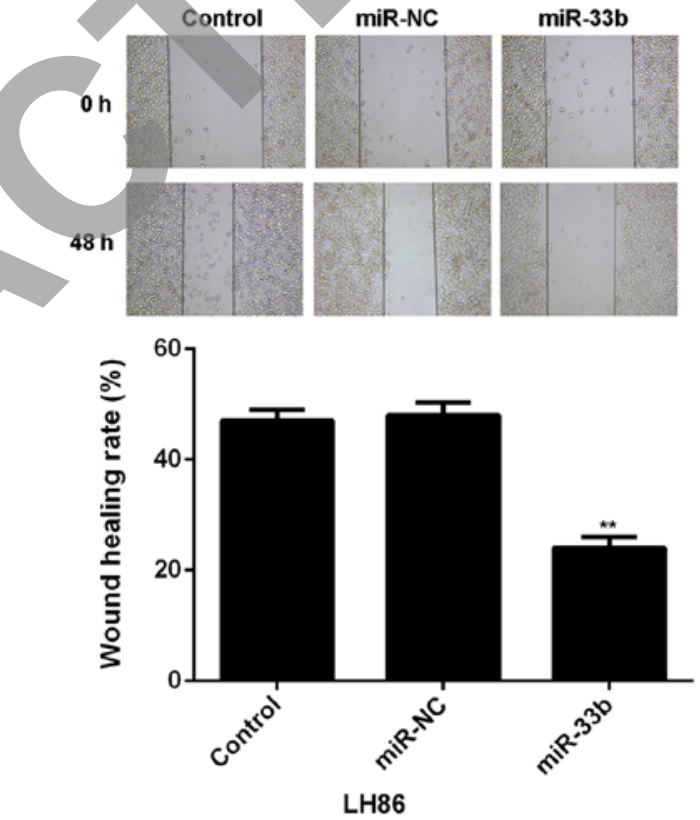

Control miR-NC miR-33b
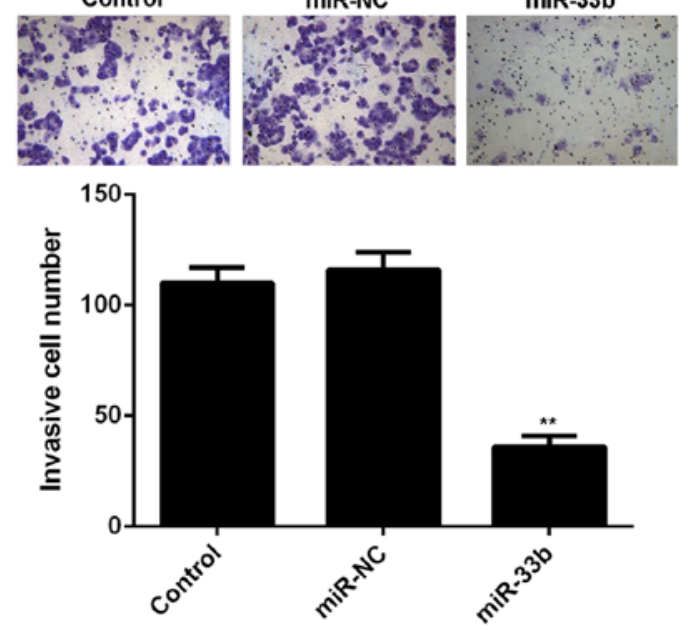

Figure 2. (A) RT-qPCR was performed to determine the relative expression of miR-33b in HepG2 and LH86 cells transfected with scramble miR (miR-NC) or miR-33b mimic.(B) MTT assay, (C) wound healing assay and (D) Transwell assay were conducted to examine cell proliferation, migration and invasion, respectively. Untransfected cells were used as controls. ${ }^{* *} \mathrm{P}<0.01$ vs. control. 
expression, we used them in our in vitro experiments to determine the role of miR-33b in regulating HCC cell proliferation, migration and invasion. As miR-33b was downregulated in HCC, a miR-33b mimic was used to transfect the HepG2 and LH86 cells in order to upregulate the expression of miR-33b. As shown in Fig. 2A, the expression level of miR-33b was decreased in the HepG2 and LH86 HCC cells following transfection with the miR-33b mimic, when compared to the control group $(\mathrm{P}<0.01)$. However, transfection with miR-NC induced no change in miR-33b levels in the HCC cells (Fig. 2A). MTT assay, wound healing assay and Transwell assay were further conducted to examine cell proliferation, migration and invasion, respectively. The overexpression of miR-33b led to a significant decrease in the proliferation, migration and invasion of the HepG2 and LH86 cells, compared to the control group $(\mathrm{P}<0.01$; Fig. $2 \mathrm{~B}-\mathrm{D})$. These data indicate that miR-33b plays a suppressive role in HCC growth and metastasis.

SALL4, a target gene of miR-33b, is negatively mediated by miR-33b in HCC cells. We then focused on the putative targets of miR-33b in HCC cells. Bioinformatics analysis was performed to predict the targets of miR-33b. As shown in Fig. 3A, SALL4 was found to be a putative target gene of miR-33b. To further clarify this prediction, we generated the luciferase vectors containing WT or MT of SALL4 3'UTR (Fig. 3B and C). Subsequently, luciferase reporter assay was conducted using the 293 cells. As shown in Fig. 3D, luciferase activity was significantly decreased in the 293 cells co-transfected with the WT SALL4 vector and miR-33b mimics, but was unaltered in the cells co-transfected with the MT SALL4 vector and miR-33b mimic, compared to the control group $(\mathrm{P}<0.01)$. These data indicate that miR-33b directly binds to the seed sequences within the SALL4 3'UTR.

As miRNAs generally inhibit the protein expression of their target genes, we then examined the effects of miR-33b overexpression or knockdown on the protein expression of SALL4 in HCC cells. The HepG2 and LH86 cells were transfected with the miR-33b mimic or miR-33b inhibitor. Transfection with the miR-33b mimic led to an upregulated miR-33b level, while transfection with miR-33b inhibitor resulted in a decrease in the miR-33b level ( $\mathrm{P}<0.01$; Fig. $3 \mathrm{E})$. We then found that the upregulation of miR-33b significantly inhibited the SALL4 protein level, while the knockdown of miR-33b expression upregulated the protein expression of SALL4 in the HepG2 and LH86 cells $(\mathrm{P}<0.01$; Fig. 3F). Therefore, we suggest that miR-33b negatively regulates the protein expression of SALL4 in HCC cells by directly binding to the 3'UTR of SALL4 mRNA.

SALL4 is involved in the miR-33b-mediated proliferation, migration and invasion of HCC cells. We further wished to determine whether SALL4 is involved in the miR-33b-mediated proliferation, migration and invasion of HCC cells. The HepG2 and LH86 cells were transfected with the miR-33b mimic with or without the SALL4 plasmid. Following transfection, MTT assay, wound healing assay and Transwell assay were further conducted to examine cell proliferation, migration and invasion, respectively. As shown in Fig. 4A-C, the proliferative, migratory and invasive capacities of the HepG2 and LH86 cells were significantly enhanced following co-transfection with the miR-33b mimic and SALL4 plasmid, when compared to the cells transfected only with the miR-33b mimic $(\mathrm{P}<0.01)$, indicating that the overexpression of SALL4 reversed the suppressive effects of miR-33b overexpression on HCC cell proliferation, migration and invasion. To further verify these findings, we examined the protein levels of SALL4 in each group. The protein level of SALL4 was higher in the HepG2 and LH86 cells co-trasnfected with the miR-33b mimic and SALL4 plasmid, when compared to the HepG2 and LH86 cells transfected only with the miR-33b mimic ( $\mathrm{P}<0.01$; Fig. 4D). These data further indicate that SALL4 is involved in the miR-33b-mediated malignant phenotypes of HCC cells.

SALL4 is upregulated in HCC and inversely correlates with $m i R-33 b$ expression. Finally, we determined the expression levels of SALL4 in HCC tissues, as well as in their matched adjacent non-tumor tissues. The results of RT-qPCR revealed that the mRNA expression of SALL4 was significantly upregulated in the HCC tissues compared to their matched adjacent non-tumor tissues $(\mathrm{P}<0.01$; Fig. $5 \mathrm{~A}$ and $\mathrm{B})$. Moreover, the SALL4 mRNA level was markedly higher in the advanced-stage HCC tissues (stages T3-T4) compared to the early-stage HCC tissues (stages T1-T2) $(\mathrm{P}<0.01)$, suggesting that the increased expression of SALL4 was significantly associated with the malignant progression of $\mathrm{HCC}$ (Fig. 5C). Furthermore, the western blot analysis data also indicated that the protein expression of SALL4 was upregulated in the HCC tissues compared to their matched adjacent non-tumor tissues ( $\mathrm{P}<0.01$; Fig. 5D). Subsequently, we further determined the expression of SALL4 in HCC cell lines. As shown in Fig. 5E and F, the mRNA and protein expression of SALL4 was also increased in the 4 HCC cell lines compared to the normal liver cells $(\mathrm{P}<0.01)$. In addition, we found that the SALL4 level inversely correlated with the miR-33b level in both HCC tissues and HCC cell lines (Fig. 5G and H). Based on these data, we suggest that miR-33b may act as a tumor suppressor in HCC through the direct inhibition of SALL4 expression.

\section{Discussion}

The role, as well as the regulatory mechanisms of miR-33b have not been previously been investigated in HCC, at least to the best of our knowledge. In the present study, we found that the expression of miR-33b was markedly reduced in HCC tissues and cell lines, when compared to the matched adjacent normal tissues and normal liver cell line. We further demonstrated that the overexpression of miR-33b suppressed the proliferation, migration and invasion of HCC cells by suppressing the protein expression of SALL4, which was significantly upregulated and inversely correlated with the miR-33b levels in HCC.

Previously, miR-33b was found to play a crucial role in controlling cholesterol and lipid metabolism in concert with their host genes, the sterol-regulatory element-binding protein (SREBP) transcription factors (22). The deregulations of miR-33b have gradually been reported in several types of human cancer. For example, Lv et al reported that the deletion, amplification or mutation at the precursor miR-33b was detected in $10 \%$ of medulloblastomas (23). Moreover, miR-33b and carnitine O-palmitoyltransferase type I were found to be significantly downregulated in gastric carcinoma, suggesting that the downregulation of miR-33b may be mediated by conditions that also affect the expression of lipogenic gene-related transcription factors (24). miR-33b has also been shown to be 
A

\begin{tabular}{|c|c|}
\hline 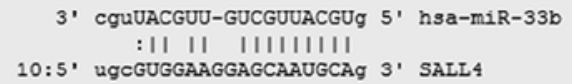 & $\begin{array}{ll}\text { mirSVR score: } & -0.2035 \\
\text { PhastCons score: } 0.4592\end{array}$ \\
\hline
\end{tabular}

B

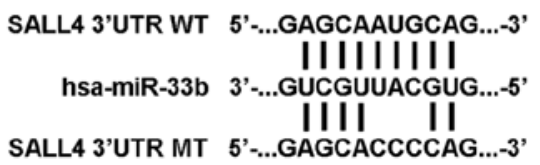

D
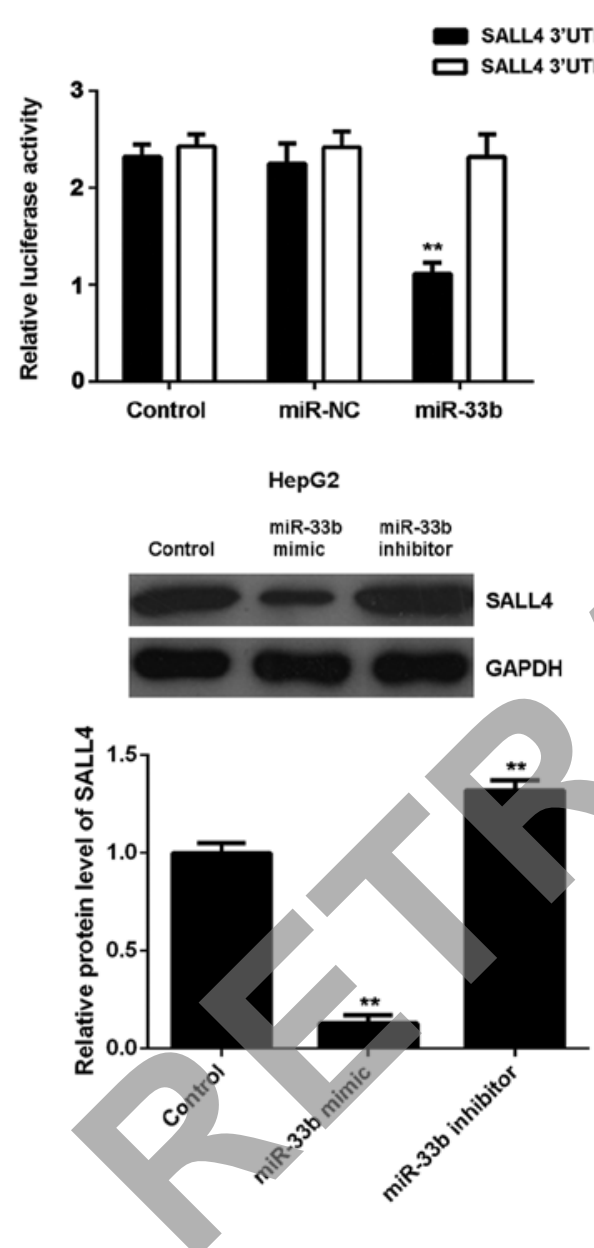

C

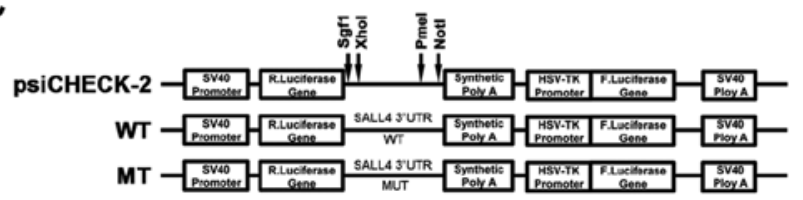

$\mathbf{E}$

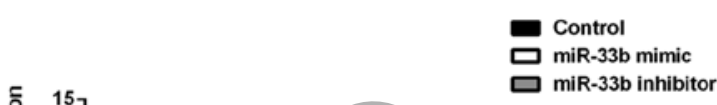

Figure 3. (A) TargetScan software predicted that Sal-like protein 4 (SALL4) was a target of miR-33b. (B) The seed sequences of miR-33b in the wild-type (WT) or mutant type (MT) of the SALL4 3' untranslated region (3'UTR) are indicated. (C) The WT or MT SALL4 3'UTR were cloned into luciferase reporter vector. (D) The luciferase activity was significantly decreased in 293 cells co-transfected with the WT SALL4 vector and miR-33b mimic, but was unaltered in 293 cells co-transfected with the MT SALL4 vector and miR-33b mimics, compared to the control group. Control: 293 cells only transfected with WT SALL4 or MT SALL4 vectors. NC: 293 cells co-transfected with WT SALL4 or MT SALL4 vector and scramble miR. ${ }^{* * *} \mathrm{P}<0.01$ vs. control. (E) miR-33b expression was examined in HepG2 and LH86 cells transfected with miR-33b mimic or miR-33b inhibitor, and (F) the protein level of SALL4 was then examined by western blot analysis. Untransfected cells were used as controls. ${ }^{* *} \mathrm{P}<0.01$ vs. control.

downregulated in multiple myeloma, and to be associated with disease progression and poor prognosis $(25,26)$. In the present study, we demonstrated that miR-33b was significantly downregulated in $\mathrm{HCC}$ tissues and cell lines, and played a suppressive role in the regulation of $\mathrm{HCC}$ cell proliferation, migration and invasion. Further investigations are required however, to focus on the association between miR-33b expression and the clinical characteristics of patients with HCC.

As miRNAs negatively mediate the protein expression of their target genes (27), we further studied the putative targets of miR-33b. Luciferase reporter assay revealed that SALL4 was a direct target gene of miR-33b in HCC cells, and its expression was negatively mediated by miR-33b. Recently, SALL4 has been reported to be significantly upregulated in HCC and to play an oncogenic role in the extensive network of heterogeneous cellular pathways underlying hepatocarcinogenesis, suggesting that the blockade of the oncogenic role of SALL4 confers therapeutic potential in SALL4-positive HCC (28). In the present study, we also found that SALL4 was significantly upregulated in HCC tissues and cell lines, 

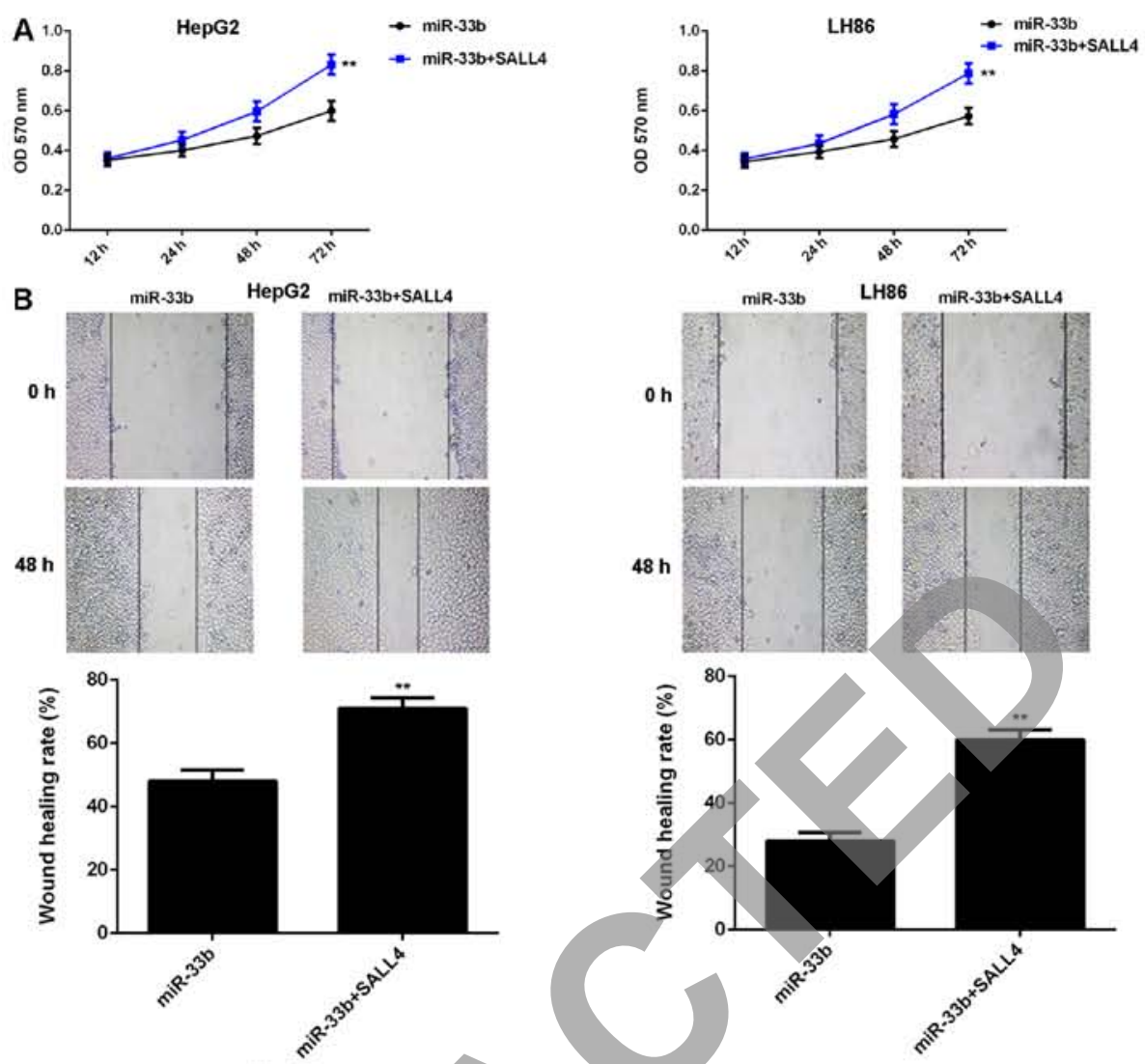

C

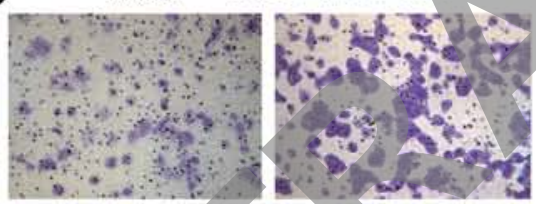

miR-33b

LH86 miR-33b+SALL4
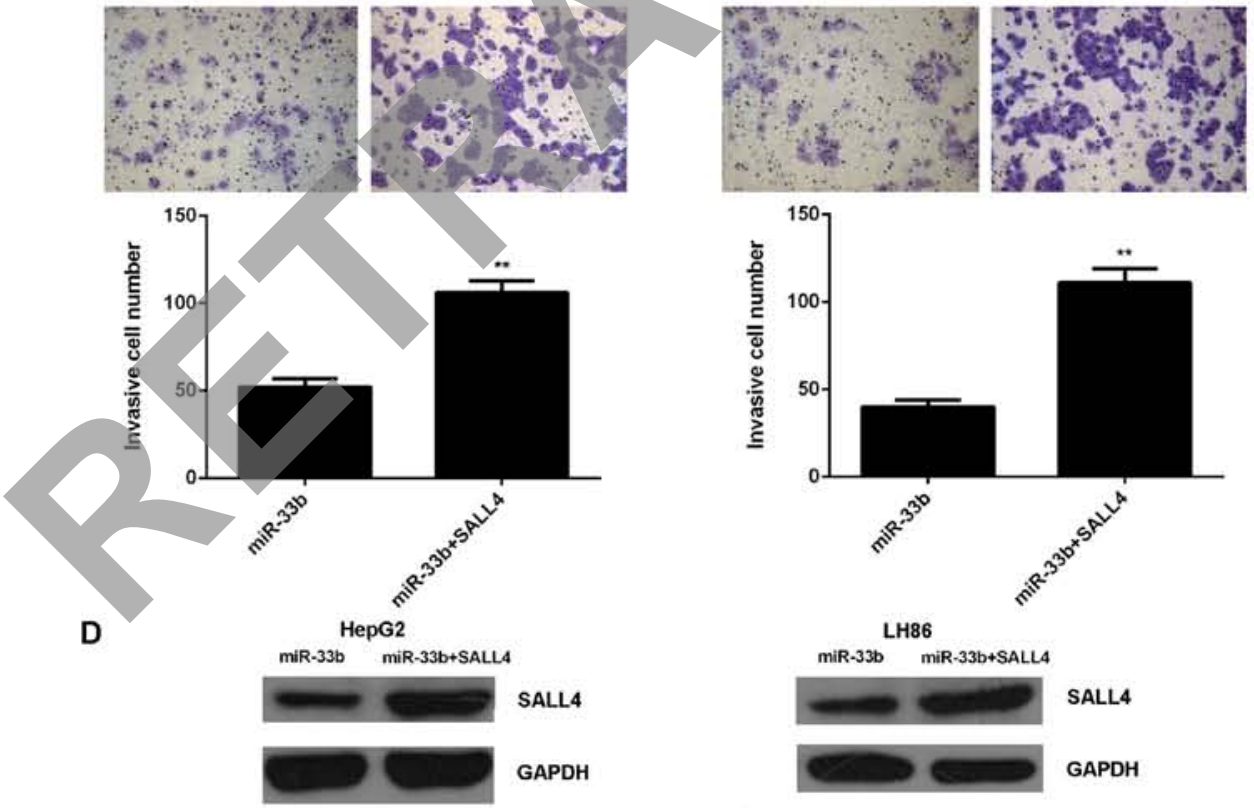

LH86

miR-33b miR-33b+SALLA
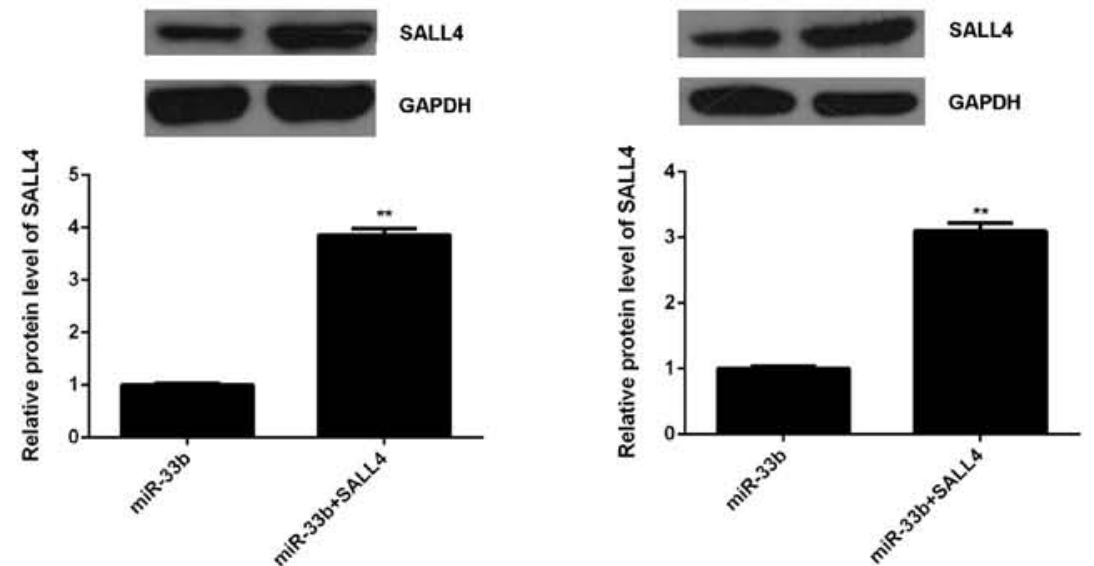

Figure 4. HepG2 and LH86 cells were transfected with miR-33b mimic with or without Sal-like protein 4 (SALL4) plasmid. Following transfection, (A) MTT assay, (B) wound healing assay and (C) Transwell assay were conducted to examine cell proliferation, migration and invasion, respectively. (D) The protein level of SALL4 was then examined by western blot analysis. ${ }^{* *} \mathrm{P}<0.01$ vs. transfection with miR-33b mimic only. 
A

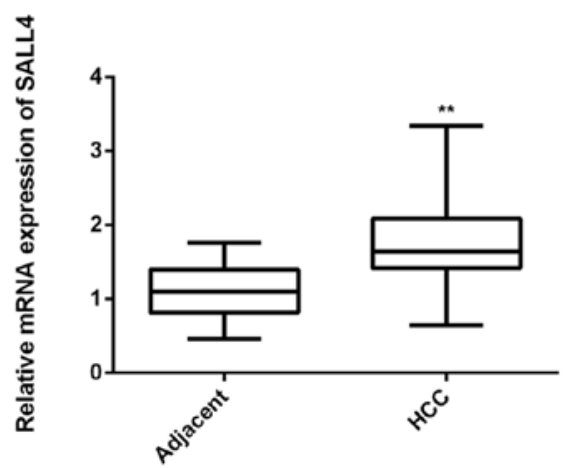

C

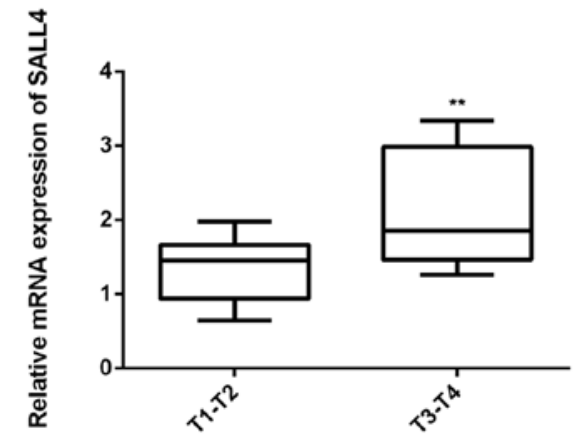

$\mathrm{E}$

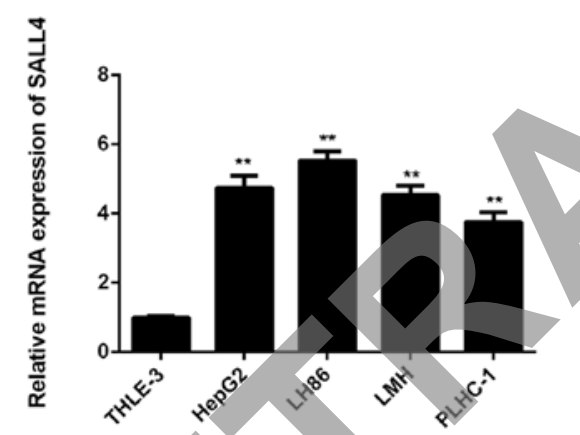

B

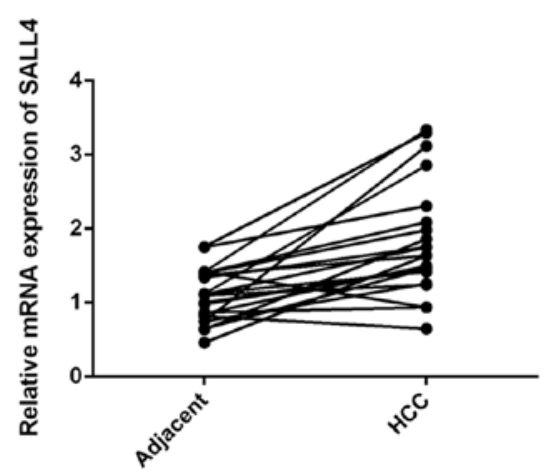

D

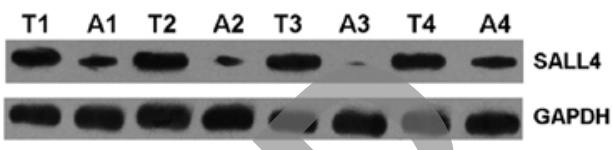

$\mathbf{F}$

THLE-3 HepG2 LH86 LMH PLHC-1

SALL4

GAPDH

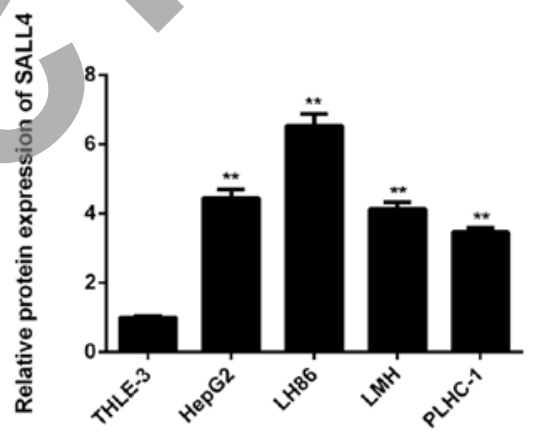

$R^{2}=0.5399$

G

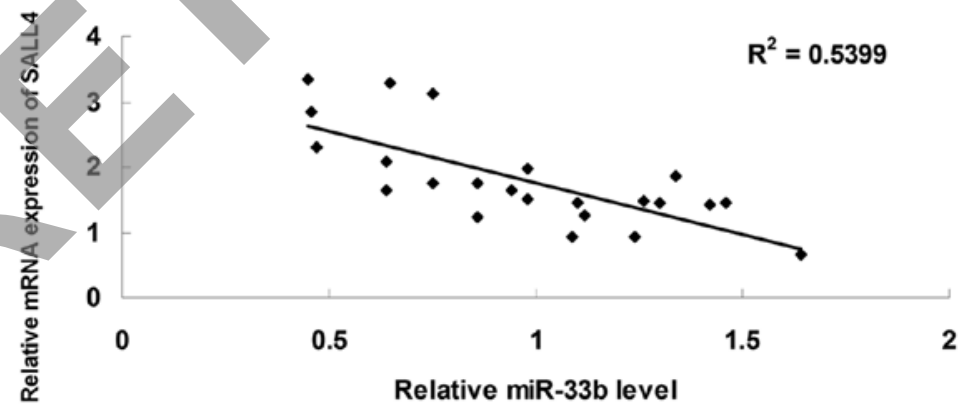

H

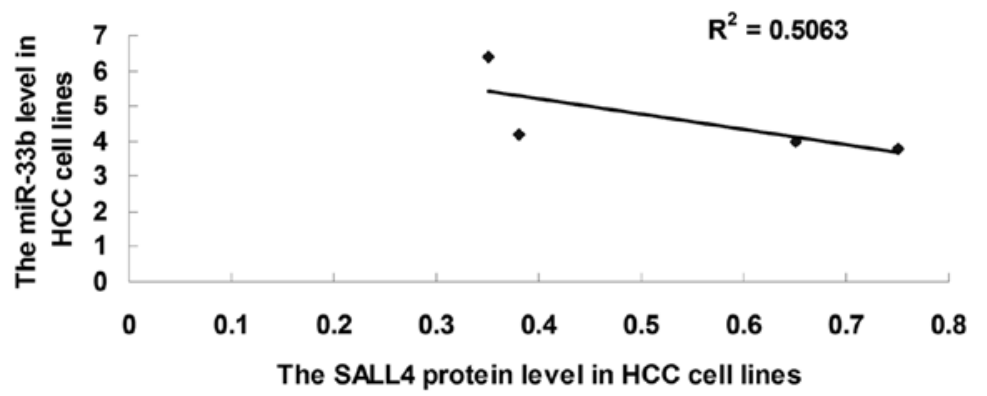

Figure 5. (A and B) RT-qPCR was used to determine the mRNA expression of Sal-like protein 4 (SALL4) in hepatocellular carcinoma (HCC) tissues and their matched adjacent non-cancerous tissues (adjacent). ${ }^{* *} \mathrm{P}<0.01$ vs. adjacent tissues. (C) RT-qPCR was used to determine the relative mRNA expression of SALL4 in the early-stage HCC tissues (stages T1-T2) and advanced-stage HCC tissues (stages T3-T4). * P<0.01 vs. stages T1-T2. (D) Western blot analysis was used to determine the protein expression of SALL4 in HCC tissues (tumor; T) and their matched adjacent non-cancerous tissues (adjacent; A). Four representative pairs are shown (A1-A4 and T1-T4). (E and F) RT-qPCR and western blot analysis were used to determine the mRNA and protein expression of SALL4 in human HCC cell lines, HepG2, LH86, LMH and PLHC-1, and the normal liver cell line, THLE-3. * ${ }^{* *}<0.01$ vs. THLE-3 cells. (G and H) The correlation between the expression levels of miR-33b and SALL4 was determined in HCC tissues and HCC cell lines. 
when compared to their matched adjacent normal tissues and normal liver cell line, respectively. Our data are consistent with those of previous studies $(11,29,30)$. Moreover, in this study, to the best of our knowledge, we demonstrated for the first time that the overexpression of SALL4 reversed the suppressive effects of miR-33b overexpression on HCC cell proliferation, migration and invasion, indicating that SALL4 is involved in the miR-33b-meditated malignant phenotypes of HCC cells. Moreover, the upregulation of SALL4 was found to inversely correlate with the downregulation of miR-33b in HCC tissues and cell lines.

Apart from SALL4, several other targets of miR-33b have also been identified in other types of cancer. For instance, Lin et al reported that miR-33b inhibited the metastasis of breast cancer cells by targeting HMGA2, SALL4 and Twistl (31). Zhang et al found that cordycepin inhibited melanoma cell invasion by upregulating miR-33b, which further led to a significant decrease in the expression of HMGA2, Twist1 and ZEB1 (32). Moreover, several signaling pathways have been implicated in the SALL4-mediated malignant phenotypes of HCC cells, including AKT, ERK MAPK and PKM2 $(33,34)$. Accordingly, we suggest that miR-33b may also participate in the regulation of these above-mentioned signaling pathways, and this needs to be verified in future studies.

In conclusion, this study demonstrates that miR-33b, upregulated in HCC, acts as a tumor suppresser in HCC by suppressing cell proliferation, migration and invasion through the inhibition of SALL4 expression. This suggests that miR-33b/SALL4 may prove to be a potential therapeutic target for $\mathrm{HCC}$

\section{References}

1. Siegel R, Naishadham D and Jemal A: Cancer statistics, 2013. CA Cancer J Clin 63: 11-30, 2013.

2. Zhu AX: Molecularly targeted therapy for advanced hepatocellular carcinoma in 2012: Current status and future perspectives. Semin Oncol 39: 493-502, 2012.

3. Dongiovanni P, Romeo S and Valenti L: Hepatocellular carcinoma in nonalcoholic fatty liver: Role of environmental and genetic factors. World J Gastroenterol 20: 12945-12955, 2014.

4. Su CH, Lin Y and Cai L: Genetic factors, viral infection, other factors and liver cancer: An update on current progress. Asian Pac J Cancer Prev 14. 4953-4960, 2013.

5. Chen C and Wang G: Mechanisms of hepatocellular carcinoma and challenges and opportunities for molecular targeted therapy. World J Hepatol 7: 1964-1970, 2015

6. Chen X, Vega VB and Ng HH: Transcriptional regulatory networks in embryonic stem cells. Cold Spring Harb Symp Quant Biol 73: 203-209, 2008

7. Zhang X, Yuan X, Zhu W, Qian H and Xu W: SALL4: An emerging cancer biomarker and target. Cancer Lett 357: 55-62, 2015.

8. Oishi N, Yamashita T and Kaneko S: Molecular biology of liver cancer stem cells. Liver Cancer 3: 71-84, 2014.

9. Oikawa T, Kamiya A, Zeniya M, Chikada H, Hyuck AD, Yamazaki Y, Wauthier E, Tajiri H, Miller LD, Wang XW, et al: Sal-like protein 4 (SALL4), a stem cell biomarker in liver cancers. Hepatology 57: 1469-1483, 2013.

10. Shikauchi Y, Saiura A, Kubo T, Niwa Y, Yamamoto J, Murase Y and Yoshikawa H: SALL3 interacts with DNMT3A and shows the ability to inhibit $\mathrm{CpG}$ island methylation in hepatocellular carcinoma. Mol Cell Biol 29: 1944-1958, 2009.

11. Zeng SS, Yamashita T, Kondo M, Nio K, Hayashi T, Hara Y, Nomura Y, Yoshida M, Hayashi T, Oishi N, et al: The transcription factor SALL4 regulates stemness of EpCAM-positive hepatocellular carcinoma. J Hepatol 60: 127-134, 2014.

12. Han SX, Wang JL, Guo XJ, He CC, Ying X, Ma JL, Zhang YY, Zhao Q and Zhu Q: Serum SALL4 is a novel prognosis biomarker with tumor recurrence and poor survival of patients in hepatocellular carcinoma. J Immunol Res 2014: 262385, 2014.
13. Ambros V: The functions of animal microRNAs. Nature 431: 350-355, 2004.

14. Tessitore A, Cicciarelli G, Del Vecchio F, Gaggiano A, Verzella D, Fischietti M, Vecchiotti D, Capece D, Zazzeroni F and Alesse E: MicroRNAs in the DNA Damage/Repair Network and Cancer. Int J Genomics 2014: 820248, 2014.

15. Esquela-Kerscher A and Slack FJ: Oncomirs - microRNAs with a role in cancer. Nat Rev Cancer 6: 259-269, 2006.

16. Coulouarn C, Factor VM, Andersen JB, Durkin ME and Thorgeirsson SS: Loss of miR-122 expression in liver cancer correlates with suppression of the hepatic phenotype and gain of metastatic properties. Oncogene 28: 3526-3536, 2009.

17. Furuta M, Kozaki KI, Tanaka S, Arii S, Imoto I and Inazawa J: miR-124 and miR-203 are epigenetically silenced tumor-suppressive microRNAs in hepatocellular carcinoma. Carcinogenesis 31: 766-776, 2010.

18. Wang W, Zhao LJ, Tan YX, Ren H and Qi ZT: MiR-138 induces cell cycle arrest by targeting cyclin D3 in hepatocellular carcinoma. Carcinogenesis 33: 1113-1120, 2012.

19. Yin W, Zhao Y, Ji YJ, Tong LP, Liu Y, He SX and Wang AQ: Serum/plasma microRNAs as biomarkers for HBV-related hepatocellular carcinoma in China. BioMed Res Int 2015: 965185, 2015.

20. Fang Y, Feng Y, Wu T, Srinivas S, Yang W, Fan J, Yang C and Wang S: Aflatoxin B1 negatively regulates Wnt/ $\beta$-catenin signaling pathway through activating miR-33a. PLoS One 8: e73004,2013

21. Antaramian A, González-Gallardo A, García-Ugalde C, Portillo W and Paredes RG: Steroid Receptors and Aromatase Gene Expression in Different Brain Areas of Copulating and Sexually Sluggish Male Rats. J Sex Med 12: 2267-2275, 2015.

22. Rottiers V and Näär AM: MicroRNAs in metabolism and metabolic disorders. Nat Rev Mol Cell Biol 13: 239-250, 2012.

23. Lv SQ, Kim YH, Giulio F, Shalaby T, Nobusawa S, Yang H, Zhou Z, Grotzer $M$ and Ohgaki $\mathrm{H}$ : Genetic alterations in microRNAs in medulloblastomas. Brain Pathol 22: 230-239, 2012.

24. Miyachi K, Sawada Y, Shida Y, Sugawara A and Hisatomi H: Lipogenic gene expression profile in patients with gastric cancer. Mol Clin Oncol 1: 825-827, 2013.

25. Hao M, Zang M, Wendlandt E, Xu Y, An G, Gong D, Li F, Qi F, Zhang, Yang Y, et al: Low serum miR-19a expression as a novel poor prognostic indicator in multiple myeloma. Int J Cancer 136: 1835-1844, 2015

26. Li F, Hao M, Feng X, Zang M, Qin Y, Yi S, Li Z, Xu Y, Zhou L, Sui $\mathrm{W}$, et al: Downregulated miR-33b is a novel predictor associated with disease progression and poor prognosis in multiple myeloma. Leuk Res 39: 793-799, 2015.

27. Yoshitaka T, Kawai A, Miyaki S, Numoto K, Kikuta K, Ozaki T, Lotz $\mathrm{M}$ and Asahara $\mathrm{H}$ : Analysis of microRNAs expressions in chondrosarcoma. J Orthop Res 31: 1992-1998, 2013.

28. Yong KJ, Gao C, Lim JS, Yan B, Yang H, Dimitrov T, Kawasaki A, Ong CW, Wong KF, Lee S, et al: Oncofetal gene SALL4 in aggressive hepatocellular carcinoma. N Engl J Med 368: 2266-2276, 2013

29. Park H, Lee H, Seo AN, Cho JY, Choi YR, Yoon YS, Han HS, Park YN and Kim H: SALL4 Expression in Hepatocellular Carcinomas Is Associated with EpCAM-Positivity and a Poor Prognosis. J Pathol Transl Med 49: 373-381, 2015.

30. Shibahara J, Ando S, Hayashi A, Sakamoto Y, Hesegawa K, Kokudo N and Fukayama M: Clinicopathologic characteristics of SALL4-immunopositive hepatocellular carcinoma. Springerplus 3: 721, 2014.

31. Lin Y, Liu AY, Fan C, Zheng H, Li Y, Zhang C, Wu S, Yu D, Huang Z, Liu F, et al: MicroRNA-33b Inhibits Breast Cancer Metastasis by Targeting HMGA2, SALL4 and Twist1. Sci Rep 5: 9995, 2015.

32. Zhang P, Huang C, Fu C, Tian Y, Hu Y, Wang B, Strasner A, Song Y and Song E: Cordycepin (3'-deoxyadenosine) suppressed HMGA2, Twist1 and ZEB1-dependent melanoma invasion and metastasis by targeting miR-33b. Oncotarget 6: 9834-9853, 2015.

33. Lee SA, Ho C, Roy R, Kosinski C, Patil MA, Tward AD, Fridlyand $\mathrm{J}$ and Chen $\mathrm{X}$ : Integration of genomic analysis and in vivo transfection to identify sprouty 2 as a candidate tumor suppressor in liver cancer. Hepatology 47: 1200-1210, 2008.

34. Wang C, Delogu S, Ho C, Lee SA, Gui B, Jiang L, Ladu S, Cigliano A, Dombrowski F, Evert M, et al: Inactivation of Spry2 accelerates AKT-driven hepatocarcinogenesis via activation of MAPK and PKM2 pathways. J Hepatol 57: 577-583, 2012. 